\title{
Four new species of the genus Eurytenes Foerster (Hymenoptera: Braconidae: Opiinae) from China
}

\author{
Qiong Wu \& Xue-xin Chen
}

Wu, Q. \& Chen, X. 2005: Four new species of the genus Eurytenes Foerster (Hymenoptera: Braconidae: Opiinae) from China. — Entomol. Fennica 16:225-232.

The species of Eurytenes Foerster in China are revised and four new species are described from China: E. basinervis sp. n., E. glabratus sp. n., E. setoputeus sp.n. and E. rugosulcus sp. n. This genus is recorded from China for the first time.

Q. Wu, Institute of Applied Entomology, Zhejiang University, Hangzhou 310029 , China; qiong0119@sina.com

X. Chen, Institute of Applied Entomology, Zhejiang University, Hangzhou 310029, China; xxchen@zju.edu.cn

Received 18 October 2004, accepted 15 April 2005

\section{Introduction}

Eurytenes Foerster, 1862 (Hymenoptera: Braconidae) is a small cosmopolitan genus that contains six subgenera and 16 described species (Fischer 1998, Fischer \& Koponen 1999). Members of the genus contain parasitoids of Agromyzidae (Diptera), but the male and biology of the new species described in this paper are unknown.

The genus can be identified using the key to the opiine genera by Wharton (1997). This genus can also be recognized from other genera (including Opius) of the subfamily Opiinae by the following characters: vein $r$ of fore wing arising from base to extreme base of pterostigma; pterostigma long and narrow, weakly widened towards its apex; vein CU1b of fore wing mediumsized, its length at least subequal to vein 3-CU1; malar suture distinct; hypoclypeal depression present, large; mandible at most with a ventral carina; first metasomal tergite slender and its dorsope often minute or obsolescent; cu-a straight and slightly reclivous.

Two subgenera, viz. Eurytenes and Stigmatopoea, are involved in this paper. In the subgenus Eurytenes, two species are known: E. abnormis
(Wesmael, 1835) from Europe and North America, and E. orientalis Fischer, 1966 from the Philippines. Stigmatopoea includes five known species from the Nearctic, Palaearctic and Oriental Regions. During the course of a study on Chinese Opiinae, we examined all the opiine specimens in the main insect collections in China, including the collections of the Chinese Academy of Sciences both in Beijing and Shanghai, and in our own institution. Only a few specimens of the genus were present, and four new species are described in this paper. These species are from the East Palaeartic and Oriental part of China. This genus is recorded from China for the first time.

The terminology used follows van Achterberg (1993). The type and other material are deposited in the parasitic Hymenoptera collection, Zhejiang University, Hangzhou, China (ZJUH).

\section{Descriptions of new species}

Eurytenes basinervis sp. n. (Fig. 1)

Material examined. Holotype + , China: Fengyangshan, Zhejiang province, 12.VIII.1984, Lirong Shen, No. 843501. 

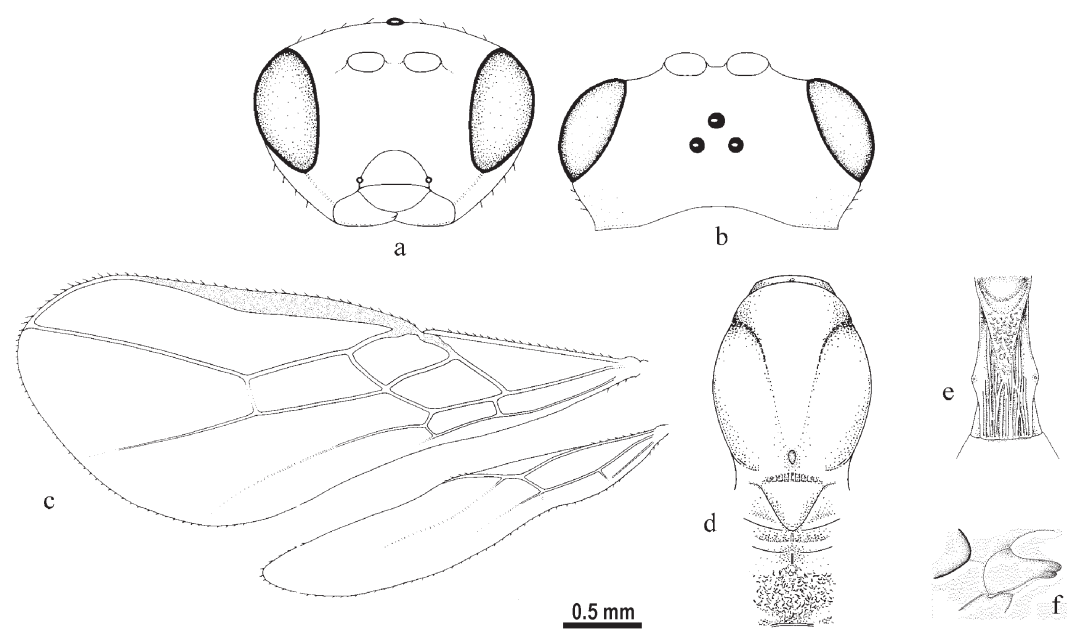

Fig. 1. Eurytenes basinervis sp. n., holotype q. - a. Head, frontal aspect. - b. Head, dorsal aspect. - c. Wings. - d. Mesosoma, dorsal aspect. - e. First metasomal tergite, dorsal aspect. F. Mandible, ventro-lateral aspect. Scale bar: $a-b-1.5 \times$; c- $0.8 \times ; d-1.0 \times ; e-$ $1.3 \times ; f-3 \times$.

Description. Length of body $3.1 \mathrm{~mm}$, length of fore wing $3.7 \mathrm{~mm}$.

Head. Antenna 1.4 times as long as fore wing, with 40 segments, length of third segment 0.9 times fourth segment, length of third, fourth and penultimate segments 2.7, 3.0 and 3.5 times their width, respectively; scapus not compressed, ventral side with dense setae, remainder with shorter and sparser setae; length of maxillary palp 1.2 times height of head, with 6 segments; OOL : diameter of posterior ocellus : $\mathrm{POL}=3.5: 1.3: 1$; frons glabrous; length of eye in dorsal view 1.6 times temple; vertex and temple smooth and sparsely setose; temples subparallel-sided behind eyes; occipital carina present up to dorsal level of eye in lateral view; face with dense, long setae; anterior tentorial pits distinct and middle-sized; clypeus punctate, slightly concave ventrally, in lateral view distinctly removed from mandibles; in frontal view hypoclypeal depression middlesized and deep; length of malar space 1.3 times basal width of mandible; mandible normal, twisted apically, and with a ventral carina near to and a little ventrad of the base of occipital carina.

Mesosoma. Length of mesosoma 1.3 times as long as its height; pronope small and deep; propleuron flattened, longitudinally punctate mediolaterally; side of pronotum punctate postero-dorsally and with long setae; precoxal sulcus deep, smooth, not reaching base of middle coxa; remainder of mesopleuron glabrous except for anterior-dorsally, and epicnemial area crenulate; pleural sulcus without crenulation; postpectal ca- rina absent; metapleuron glabrous dorsally, densely setose and irregularly rugose ventrally; mesosternal sulcus deep and distinctly crenulate; notauli deep, crenulate and setose on its anterior $1 / 3$, its posterior $2 / 3$ obsolescent and smooth, mid-posterior depression deep; mesoscutum smooth; scutellar sulcus wide, deep and crenulate; scutellum rather convex, smooth and setose posteriorly; propodeum irregularly sculptured, without median carina.

Wings. Fore wing: pterostigma long and narrow, slightly widened towards its apex; $r$ arising from base of pterostigma; 1-M straight; $\mathrm{r}$ : 3-SR : $\mathrm{SR} 1=1.0: 1.9: 4.0 ; 1-\mathrm{SR}+\mathrm{M}$ distinctly sinuate; SR1 nearly straight and ending close to apex of wing; cu-a vertical, straight; 1 -CU1 : 2-CU1 = 1.0 : 3.9; 2-SR : 3 -SR : $r-m=1.5: 3.1: 1.0$; first subdiscal cell rather robust and widened apically; CU1b longer than 3CU1; m-cu just postfurcal, and parallel to 1-M posteriorly. Hind wing: SR as an unpigmented fold but distinct basally; m-cu long and straight; 1-M longer than 1r-m; $\mathrm{M}+\mathrm{CU}$ : $1-\mathrm{M}=1.3: 1$.

Legs. Hind coxa sparsely setose; tarsal claws normal, sparsely setose and without lobe; length of femur, tibia and basitarsus of hind leg 4.1, 7.8 and 5.0 times as long as their width, respectively.

Metasoma. Length of first tergite 1.8 times its apical width, its surface longitudinally sculptured, shallowly rugose medially and slightly concave basally, its dorsal carinae strong basally, not reaching apex of tergite; spiracle of first tergite small; laterope large and deep; dorsope pres- 
ent because of strong surrounding carina giving its deep impression of presence; second tergite smooth and sparsely setose; ovipositor straight, apically acute, length of ovipositor sheath 0.2 times length of fore wing and 1.4 times first metasomal tergite; sheath sparsely setose.

Colour. Dark brown; scapus of antenna, mandible, propleuron ventrally, middle and hind coxae, all femora (dorsally brown), fore and middle tibiae, and ovipositor yellowish brown; maxillary and labial palpi, fore coxa, all trochanters, fore and middle tarsi brownish yellow; pterostigma, veins, hind tibia and tarsus, metasoma (but median part of each tergite brownish yellow) brown; wing membrane subhyaline.

Notes. This species is closely related to $E$. orientalis Fischer, but can be separated from the latter in having the propodeum irregularly sculptured, without median carina, and the precoxal sulcus deep, smooth, not reaching the base of middle coxa (Fischer 1966, 1987).

\section{Eurytenes glabratus sp. n. (Fig. 2)}

Material examined. Holotype + , China: Jinding, Fanjingshan, Guizhou province, 12.VII.1993, Song-lin Yao, No. 936904. Paratype: 1 q, same locality as holotype, 14.VII.1993, Xue-xin Chen, No. 939436.

Description. Length of body $2.8 \mathrm{~mm}$, length of fore wing $3.4 \mathrm{~mm}$.

Head. Antenna 1.6 times as long as fore wing, with 38 segments, length of third segment 1.0 times fourth segment, length of third, fourth and penultimate segments $4.2,4.0$ and 3.3 times as long as their width, respectively; scapus slightly compressed, densely setose; length of maxillary palp 1.4 times as long as height of head, with 6 segments; OOL : diameter of posterior ocellus : $\mathrm{POL}=2.5: 1.2: 1.0$; frons glabrous; length of eye in dorsal view 1.9 times temple; vertex smooth, sparsely setose; temple smooth, sparsely setose, temples subparallel-sided behind eyes; occipital carina present up to $2 / 3$ level of eye; face setose and finely punctate, with short medio-longitudinal carina; anterior tentorial pits rather small and distinct; clypeus finely punctate, slightly concave ventrally, in lateral view distinctly removed from mandibles; in frontal view hypoclypeal depression distinct; length of malar space 0.9 times as
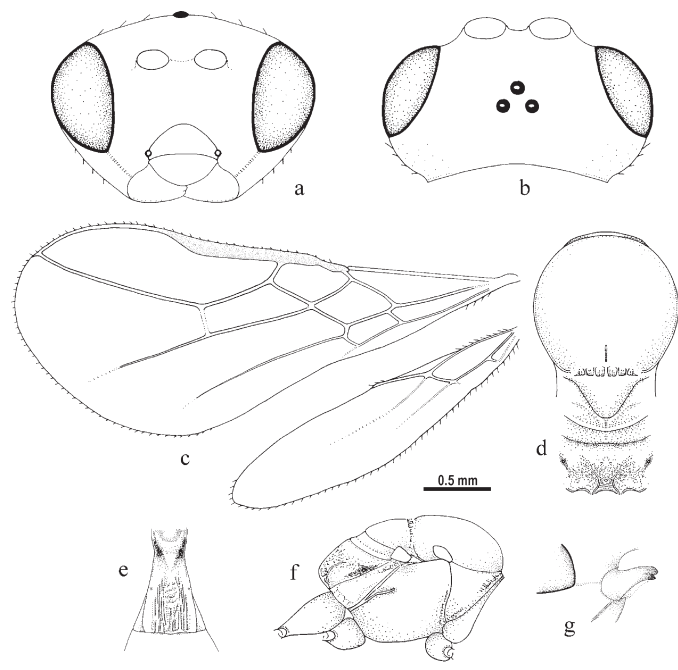

Fig. 2. Eurytenes glabratus sp. n., holotype.$+-a$. Head, frontal aspect. - b. Head, dorsal aspect. - c. Wings. - d. Mesosoma, dorsal aspect. - e. Mesosoma, lateral aspect. - f. First metasomal tergite, dorsal aspect. $-\mathrm{g}$. Mandible, ventro-lateral aspect. Scale bar: $a-b-2 \times ; c-0.8 \times ; d-1.4 \times ; e-1.0 \times ; f-1.5 \times$; $g-2.3 \times$.

long as basal width of mandible; mandible normal, twisted apically, and with a ventral carina near to and a little ventrad of the base of occipital carina.

Mesosoma. Length of mesosoma 1.5 times as long as its height; pronope obsolescent; propleuron flattened; side of pronotum smooth except for some short crenulae antero-ventrally and antero-dorsally; precoxal sulcus absent; mesopleuron smooth, sparsely setose; pleural sulcus without crenulation; postpectal carina absent; metapleuron dorsally smooth with rather sparse, long setae and posteriorly rugose; mesosternal sulcus deep, distinctly crenulate; notauli absent, only with shallow longitudinal medio-posterior depression; mesoscutum smooth, sparsely setose; scutellar sulcus narrow, deep and crenulate; scutellum rather convex, smooth; propodeum without median carina, comparatively smooth on its median part, irregularly rugose posteriorly, slightly concave latero-apically, densely setose medially; propodeal spiracle round and small.

Wings. Fore wing: pterostigma long and narrow, slightly widened towards its apex; $r$ arising from basal third of pterostigma; 1-M straight, 
hardly curved posteriorly; $r$ : $3-\mathrm{SR}: \mathrm{SR} 1=1: 3.4$ : $8.0 ; 1-\mathrm{SR}+\mathrm{M}$ distinctly sinuate; SR1 nearly straight and ending close to apex of wing; submarginal cell comparatively large; cu-a subvertical, straight; 1 -CU1 : 2 -CU1 = $1.0: 4.2 ; 2-\mathrm{SR}$ $: 3-\mathrm{SR}: \mathrm{r}-\mathrm{m}=2.3: 2.5: 1.0$; first subdiscal cell rather robust; CU1b medium-sized, subequal to $3 \mathrm{CU} 1$; m-cu just postfurcal, and parallel to 1-M posteriorly. Hind wing: SR largely absent, only as an unpigmented fold; $\mathrm{m}$-cu straight; 1-M longer 1r-m; $\mathrm{M}+\mathrm{CU}: 1-\mathrm{M}=1.0: 2.0$; cu-a straight and slightly converging to base of hind wing.

Legs. Hind coxa setose; tarsal claws normal, setose and without lobe; length of femur, tibia and basitarsus of hind leg 7.0, 11.3 and 5.8 times as long as their width, respectively.

Metasoma. Length of first tergite 1.7 times as long as its apical width, its surface comparatively smooth laterally, transversely rugose medially, longitudinally sculptured medio-posteriorly and concave basally, its dorsal carinae strong, distinct basally, not reaching apex of tergite; spiracle of first tergite small; laterope medium-sized; dorsope small and deep; ovipositor slightly curved, apically acute; ovipositor sheath ribbonshaped; length of ovipositor sheath 0.1 times length of fore wing and 1.1 times as long as first metasomal tergite.

Colour. Dark brown; ventral margin of clypeus, mandible, middle and hind coxae, trochanters, femora ventrally and ovipositor brownish yellow; maxillary palp, labial palp and fore coxa yellow; femora dorsally, tibiae (basally brownish yellow) and tarsi brown; posterior margin of third tergite yellowish brown; wing membrane subhyaline; pterostigma and veins brown (but veins of hind wing pale).

Notes. This species belongs to the subgenus Stigmatopoea Fischer, 1998 of the genus Eurytenes by the characters of its clypeus, mandible and precoxal sulcus, but can be separated from the other described species in this subgenus by having the antenna with 38 segments, the vein $\mathrm{m}-\mathrm{cu}$ of fore wing postfurcal, the vein 2-SR+M and submarginal cell of fore wing comparatively short, the precoxal sulcus smooth; the notauli absent, only with a narrow shallow and longitudinal mid-posterior depression; and the propodeum irregularly rugose posteriorly (Fischer 1959, 1972).

\section{Eurytenes setoputeus sp. n. (Fig. 3)}

Material examined. Holotype + , China: Jinding, Fanjingshan, Guizhou province, 13.VII.1993, Xuexin Chen, No. 938801.

Description. Length of body $2.7 \mathrm{~mm}$, length of fore wing $3.5 \mathrm{~mm}$.

Head. Antenna 1.6 times as long as fore wing, with 38 segments, length of third segment 1.1 times fourth segment, length of third, fourth and penultimate segments 4.4, 4.0 and 3.0 times as long as their width, respectively; scapus normal, its inner side more densely setose than its outer side; length of maxillary palp as long as height of head, with 6 segments; OOL : diameter of posterior ocellus : $\mathrm{POL}=3.5: 1.0: 1.0$; frons glabrous; length of eye in dorsal view 2 times as long as temple; vertex and temple sparsely setose; temples parallel-sided behind eyes; occipital carina present, exceeding dorsal margin of eyes, shortly absent near the base of mandible; face smooth, setose and convex, inner side of eyes finely and densely punctate; anterior tentorial pits mediumsized; clypeus with a row of long setae above ventral margin, ventrally slightly concave, in lateral view distinctly removed from mandibles; in frontal view hypoclypeal depression moderately deep; length of malar space 0.9 times as long as basal width of mandible; mandible normal, twisted apically, and with a ventral carina separated from and a little ventrad of the base of occipital carina.

Mesosoma. Length of mesosoma 1.3 times as long as its height; pronope large, deep and ovalform; propleuron flattened, smooth, sparsely setose; side of pronotum crenulate in groove on both sides of pronope, extending to medio-anterior margin ventrally; precoxal sulcus absent; remainder of mesopleuron smooth; pleural sulcus smooth, not crenulate; postpectal carina absent; metapleuron dorsally glabrous, ventrally setose and irregularly rugose; mesosternal sulcus deep, distinct and crenulate; notauli only present at its anterior half, medio-posterior depression pit-like, surroundings setose; middle lobe of mesoscutum setose, lateral lobe glabrous; scutellar sulcus narrow, crenulate; scutellum rather convex, smooth and setose; propodeum areolate, densely punctate except for two latero-posterior areolae.

Wings. Fore wing: pterostigma long and nar- 


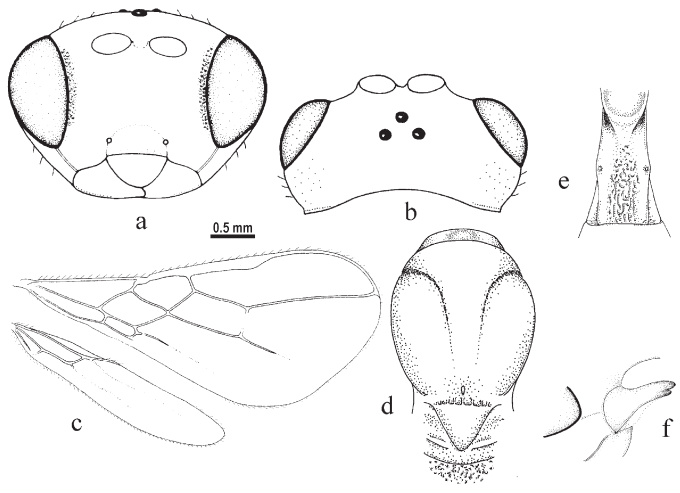

Fig. 3. Eurytenes setoputeus sp. n., holotype $9 .-$ a. Head, frontal aspect. - b. Head, dorsal aspect. - c. Wings. - d. Mesosoma, dorsal aspect. - e. First metasomal tergite, dorsal aspect. $-\mathrm{f}$. Mandible, ventro-lateral aspect. Scale bar: $a-b-3 \times ; c-1.0 \times ; d$ $-2.7 \times ; e-3 \times ; f-4 \times$.

row, slightly widened towards its apex; $r$ arising from basal third of pterostigma; 1-M straight, hardly curved posteriorly; $r$ : $3-S R$ : SR $1=1.0$ : $3.8: 4.4 ; 1-\mathrm{SR}+\mathrm{M}$ distinctly sinuate; SR1 nearly straight and ending close to apex of wing; cu-a straight, slightly converging to base of fore wing posteriorly; 1-CU1 : 2-CU1 = $1.0: 3.0 ; 2-S R: 3$ SR $: r-m=1.2: 2.0: 1.0$; first subdiscal cell rather robust and widened apically; CU1b longer than 3CU1; m-cu postfurcal, and parallel to 1-M posteriorly. Hind wing: SR only as an unpigmented fold; m-cu absent; 1-M longer than 1r-m; M+CU : $1-\mathrm{M}=1.4: 1.0$; cu-a straight and slightly converging to base of hind wing posteriorly.

Legs. Hind coxa setose; tarsal claws normal, setose; length of femur, tibia and basitarsus of hind leg 8.2, 8.1, and 5.6 times as long as their width, respectively.

Metasoma. Length of first tergite about 2.1 times as long as its apical width, its surface transversely sculptured laterally, irregularly sculptured medially and concave basally, its dorsal carinae strong basally, not reaching apex of tergite; spiracle of first tergite small, laterally situated; laterope small and deep; dorsope absent (but because of strong surrounding carina giving impression of presence); second tergite smooth; each tergite setose posteriorly; ovipositor slightly down-curved, apically acute, length of ovipositor sheath 0.1 times length of fore wing and as long as first metasomal tergite.
Colour. Dark brown; clypeus ventrally, mandible (but apically brown), scapus and pedicel ventrally, trochantellus apically, femur dorsally, and ovipositor yellowish brown; coxa, trochanter, femur ventrally, fore and middle tibiae, and tarsi (but telotarsi brown) brownish yellow; flagellum, veins, pterostigma, hind tibia and tarsus brown; maxillary and labial palpi yellow; wing membrane subhyaline.

Notes. This species is similar to E. glabratus sp. nov., but can be separated from the latter and other species in subgenus Stigmatopoea in having the face near the inner side of eye punctate; the pronope large, deep and oval-form; the notauli present on its anterior half; and the precoxal sulcus absent (Fischer 1959, 1972)

Eurytenes rugosulcus sp. n. (Fig. 4)

Material examined. Holotype + , China: Wuyishan, Fujian province, 1.V.1989, Jiashe Wang, No.964337. Paratype: 1 , same locality as holotype, 15.IX.1989, Jiashe Wang, No. 964460 .

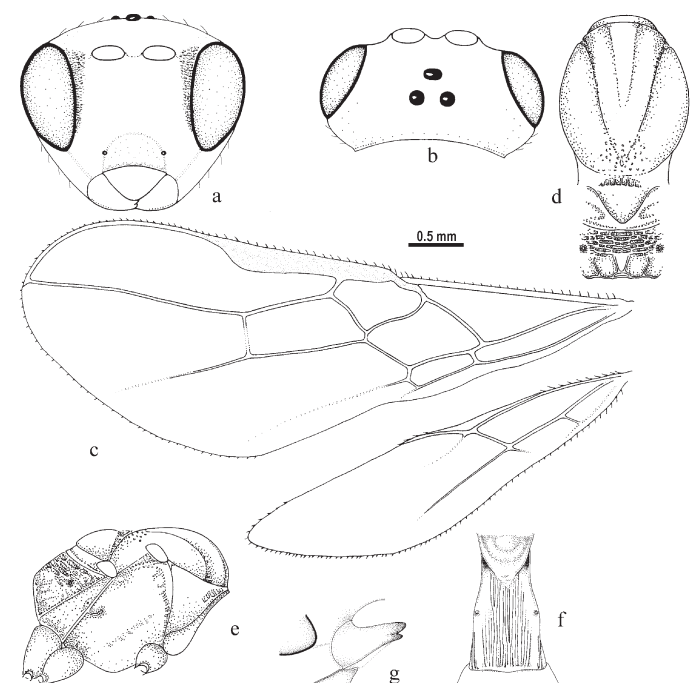

Fig. 4. Eurytenes rugosulcus sp. n., holotype $9 .-$ a. Head, frontal aspect. - b. Head, dorsal aspect. - c. Wings. - d. Mesosoma, dorsal aspect. - e. Mesosoma, lateral aspect. - f. First metasomal tergite, dorsal aspect. $-\mathrm{g}$. Mandible, ventro-lateral aspect. Scale bar: $a-b-1.8 \times ; c-1.1 \times ; d-1.3 \times ; e-1 \times ; f-1.7 \times$; $g-2.1 \times$. 
Description. Length of body $1.2 \mathrm{~mm}$, length of fore wing $3.8 \mathrm{~mm}$.

Head. Antenna 1.8 times as long as fore wing, with 47 segments, length of third segment 1.0 times fourth segment, length of third, fourth and penultimate segments $2.6,2.8$ and 4.0 times as long as their width, respectively; scapus setose; length of maxillary palp 1.8 times as long as height of head, with 6 segments; OOL : diameter of posterior ocellus : $\mathrm{POL}=5.5: 1.0: 1.0$; frons smooth, with a shallow depression behind antennal sockets; length of eye in dorsal view 1.7 times temple; vertex and temple sparsely setose, temples subparallel-sided behind eyes; occipital carina present, exceeding dorsal level of eye in lateral view; face sparsely punctate and setose, inner side of eyes densely punctate and with two rows of setae, medio-longitudinal carina widened towards epistomal suture with dense and deep punctures on its dorsal half; anterior tentorial pits small but distinct; clypeus punctate and setose, ventrally flat, in lateral view distinctly removed from mandibles; in frontal view hypoclypeal depression moderately deep; length of malar space 1.4 times as long as basal width of mandible; mandible normal, twisted apically, and with a ventral carina near to and almost at the level of the base of occipital carina.

Mesosoma. Length of mesosoma 1.4 times as long as its height; pronope obsolescent; propleuron flattened, crenulate laterally, with a shallow transverse suture dorsad of flange; side of pronotum with transverse crenulate groove on both sides of pronope; precoxal sulcus shallow and distinct, slightly punctate, not reaching base of middle coxa; remainder of mesopleuron smooth, setose dorsally and ventrally, epicnemial area crenulate; pleural sulcus finely crenulate dorsally and comparatively robust ventrally; postpectal carina absent; metapleuron setose and with robust rugae dorsally and ventrally; mesosternal sulcus normal, distinctly crenulate; notauli setose, crenulate in anterior third, smooth medially and finely punctate posteriorly, midposterior depression small and shallow; mesoscutum smooth, middle lobe of mesoscutum with a pair of grooves parallel to notauli; scutellar sulcus wide, deep and distinctly crenulate; scutellum rather convex, smooth and setose; propodeum setose, deeply and transversely sculptured on its anterior half, its posterior half areolate and comparatively smooth in each areola.

Wings. Fore wing: pterostigma long and narrow, slightly widened towards its apex; $r$ arising from basal third of pterostigma; 1-M slightly curved; $r$ : 3-SR : SR1 = $1.0: 5.5: 13.5 ; 1-\mathrm{SR}+\mathrm{M}$ distinctly sinuate; SR1 nearly straight and ending close to apex of wing; cu-a straight, converging to CU1b posteriorly; 1 -CU1 : 2 -CU1 $=1.0: 5.0 ; 2$ $\mathrm{SR}: 3$-SR $: \mathrm{r}-\mathrm{m}=1.3: 2.2: 1.0$; first subdiscal cell rather robust and slightly widened apically; CU1b medium-sized, as long as 3CU1; m-cu postfurcal and parallel to 1-M posteriorly. Hind wing: SR largely absent, only as an unpigmented fold; m-cu straight as an unpigmented fold; 1-M much longer than $1 \mathrm{r}-\mathrm{m} ; \mathrm{M}+\mathrm{CU}: 1-\mathrm{M}=1.2: 1.0$; cu-a straight and converging to the base of hind wing.

Legs. Hind coxa setose; tarsal claws normal and setose; length of femur, tibia and basitarsus of hind leg 4.9, 7.6, and 5.6 times as long as their width, respectively.

Metasoma. Length of first tergite 1.6 times as long as its apical width, its surface with longitudinal sculpture, its dorsal carinae strong basally, not reaching apex of tergite; spiracle of first tergite very small; laterope medium-sized and shallow; dorsope small and deep; second and its following tergites smooth, with one or two rows of setae posteriorly, respectively; ovipositor slightly curved, apically acute, length of ovipositor sheath 0.1 times length of fore wing and 0.9 times as long as first metasomal tergite.

Colour. Dark brown; clypeus ventrally, mandible (dark brown apically), propleural flange, pronotum antero-ventrally and ovipositor yellowish brown; scapus, pedicellus, coxa, trochanter, trochantellus (yellowish brown apically), femur (yellowish brown apically), fore and middle tibiae and tarsi (telotarsus yellowish brown) brownish yellow; flagellum, hind tibia (brownish yellow basally) and tarsus, pterostigma and veins brown; maxillary and labial palpi yellow; metasoma brown, third and its following tergites subhyaline behind their rows of setae; the setose band yellowish brown; wing membrane subhyaline.

Notes. This species is closely related to $E$. stigmaticus (Fischer), but can be separated from the latter in having the face near the inner side of 
the eye punctate; the malar space 1.4 times as long as basal width of mandible; the notauli completely present, setose, and its anterior third crenulate, smooth medially and finely punctate posteriorly; and the precoxal sulcus slightly sculptured (Fischer 1972).

\section{Key to the species of subgenera Eurytenes and Stigmatopoea of the genus Eurytenes Foerster}

1. Vein $r$ of fore wing arising from extreme base of pterostigma and about as long as vein 2-SR (Fig. 1c); metasoma brown, median part of each tergite brownish yellow; subgenus Eurytenes Foerster

- Vein $r$ of fore wing arising from the subbasal part of pterostigma and much shorter than vein 2-SR (Figs. 2c, 3c, 4c); colour of metasoma variable; subgenus Stigmatopoea Fischer

2. Antenna with 40 segments; face glabrous; precoxal sulcus smooth; ovipositor sheath distinctly surpass the apex of metasoma and its length 0.2 times length of fore wing

E. basinervis sp. n.

- Antenna with 33 segments at most; face finely punctate; precoxal sulcus crenulate; ovipositor sheath very short and not or hardly surpass the apex of metasoma

3. Propodeum with 5 areolae; length of hind femur about 6 times its width; length of first tergite 2.4 times its apical width; ovipositor sheath only a little surpass the apex of metasoma; metasoma yellow or reddish yellow; $2.5 \mathrm{~mm}$; Philippines

E. orientalis Fischer, 1966

- Propodeum rugose, without areolae; length of hind femur about 5 times its width; length of first tergite about 2 times its apical width; ovipositor sheath conceal and invisible; metasoma dark completely; 2.5-3.0 mm; Europe, North America

E. abnormis (Wesmael, 1835)

4. Vein $\mathrm{m}$-cu of fore wing antefurcal; $3.9 \mathrm{~mm}$; Switzerland

cf. phantasticus (Fischer, 1959)

- Vein m-cu of fore wing postfurcal or interstitial
5. Precoxal sulcus weakly sculptured; notauli completely present; middle lobe of mesoscutum with a pair of grooves parallel to notauli

E. rugosulcus sp.n.

- Precoxal sulcus smooth or absent; notauli at most present anteriorly; middle lobe of mesoscutum glabrous, without grooves 6

6. Antenna with 45-60 segments 7

- Antenna with 38 segments 8

7. Ovipositor sheath not surpass the apex of metasoma; 2.3-4 mm; Europe, Japan, North America

E. macrocerus (Thomson, 1895)

- Length of ovipositor sheath at least as long as first tergite; about $4 \mathrm{~mm}$; Austria

E. tutus (Fischer, 1977)

8. Pterosigma comparatively short and parallel apically at most; submarginal cell comparatively long; vein $\mathrm{m}$-cu of fore wing strongly postfurcal; antenna with 30-35 segments; ovipositor sheath short

- Pterosigma comparatively long and more or less widened apically; submarginal cell comparatively short; vein $\mathrm{m}$-cu of fore wing slightly postfurcal; antenna with 38 segments; ovipositor sheath comparatively long, about as long as first tergite

9. Propodeum rugose, comparatively smooth apically and with an indistinct and longitudinal carina; mid-posterior depression slightly longitudinal; face smooth, sometimes finely punctate; length of hind femur 5 times its width; third and its following tergites yellow posteriorly; $3.1 \mathrm{~mm}$; Hungary

E. cinctiventris (Fischer, 1959)

- Propodeum finely and weakly rugose; midposterior depression round; face weakly punctate and rugose; length of hind femur 6-7 times its width; third and its following tergites dark; 1.9-2.1 mm; Finland, Austria, Bulgaria, Japan E.cubitalis (Fischer, 1959)

10. Face near inner side of eyes punctate; notauli present on its anterior half, mid-posterior depression of mesoscutum pit-like and setose around E. setoputeus sp.n.

- Face near inner side of eyes without punctures; notauli absent; mid-posterior depression of mesoscutum at most present only as a narrow, shallow and longitudinal groove

E. glabratus sp.n. 
Acknowledgements. We wish to thank Prof. Dr. Ing. C. van Achterberg (Leiden, The Netherlands) for his critical review of an early version of the manuscript. We also thank him and Dr. M. Fischer (Vienna, Austria) for providing part of the literature. We are also grateful to Dr. Gavin Broad (Ascot, U. K.) for his valuable reviewer's comments. The project was partly supported by National Natural Science Foundation of China (NSFC, No. 30170120) and Program for New Century Excellent Talents in University (No. NCET-04-0521) to the second author.

\section{References}

Achterberg, C. van 1993: Illustrated key to the subfamilies of the Braconidae (Hymenoptera, Braconidae). Zoologische Verhandelingen 283: 1-189.

Fischer, M. 1959: Neue Opius Wesm.-Arten aus Polen (Hymenoptera, Braconidae). - Annales Zoologici (Warsaw) 18: 81-87.

Fischer, M. 1966: Revision der indo-australischen Opiinae
(Hymenoptera, Braconidae). - Series Entomologica 1: $1-167$.

Fischer, M. 1972: Hymenoptera: Braconidae (Opiinae I). — Das Tierreich 91: 1-620.

Fischer, M. 1987: Hymenoptera: Opiinae III - aethiopische, orientalische, australische und ozeanische Region. - Das Tierreich 104: 1-734.

Fischer, M. 1998: Neue taxonomische Untersuchungen ueber Madenwespen der Alten Welt mit besonderer Beruecksichtigung der Gattungen Eurytenes Foerster, Aulonotus Ashmead, Biosteres Foerster und der Untergattung Gastrosema Fischer (Hymenoptera, Braconidae: Opiinae). — Linzer Biologische Beitraege 30: 21-51.

Fischer, M. \& Koponen, M. 1999: A survey of Opiinae (Hymenoptera, Braconidae) of Finland, part 2. Entomologica Fennica 10: 129-160.

Wharton, R. A. 1997: Generic relationships of opiine Braconidae (Hymenooptera) parasitic on fruit-infesting Tephritidae (Diptera). - Contributions of the American Entomological Institute 30: 1-53. 